\title{
21-residue peptide's dynamics at and between elementary structural transitions
}

\author{
Dmitry Nerukh \\ Unilever Centre for Molecular Informatics, Department of Chemistry, Cambridge \\ University, Cambridge CB2 1EW, UK \\ Makoto Taiji \\ Computational Systems Biology Group, Advanced Science Institute, RIKEN, 61-1 \\ Onocho, Tsurumi, Yokohama, Kanagawa 230-0046, Japan
}

\begin{abstract}
Elementary conformational changes of the backbone of a 21-residue peptide A5(A3RA)3A are studied using molecular dynamics simulations in explicit water. The processes of the conformational transitions and the regimes of stationary fluctuations between them are investigated using minimal perturbations of the system. The perturbations consist of a few degrees rotation of the velocity of one of the system's atoms and keep the system on the same energy surface. It is found that (i) the system dynamics is insignificantly changed by the perturbations in the regimes between the transitions; (ii) it is very sensitive to the perturbations just before the transitions that prevents the peptide from making the transitions; (iii) the perturbation of any atom of the system, including distant water molecules is equally effective in preventing the transition. The latter implies strongly collective dynamics of the peptide and water during the transitions.
\end{abstract}

\section{Introduction}

Folding and conformational changes of peptides and proteins are predominantly determined by the dynamics of $\phi$ and $\psi$ dihedral angles of the biopolymer's backbone. The angle values are bounded by the steric restrictions of the backbone and the side chains. This is reflected in specific distributions of points on the Ramachandran $\phi-\psi$ plots where most of the points are concentrated at restricted areas characteristic to each aminoacid [1]. Dynamically this results in the behaviour when the biomolecule exhibits well separated 
periods of stationary fluctuations and quick transitions between them (Fig. $3)$.

The majority of publications on protein dynamics concentrate on the analysis of the free energy of the system. The dynamics in these works is understood as the transitions between the energy minima. Numerous investigations of proteins conformations and methods of their effective simulations are also based on the same energy picture. When the "true" dynamics, obtained from the realistic equations of motion, is studied it is mostly devoted to large scale motions of proteins. Obviously, the latter is only possible to study using severe approximations (for example, the diffusive dynamics of proteins [2]) or restrict the state of the protein to a particular conformation, for example, the native state [3]. The aim of the present work is to investigate the elementary events of the backbone conformational changes using realistic atomistic model of the system. It is also essential to include water molecules explicitly since they play crucial role in the process.

From the commonly accepted "folding funnel" point of view these changes should correspond to barrier crossing with gradual descending on the free energy surface that would eventually lead to the native state. However, it should be remembered that these major conformational transitions defined by the backbone rearrangements involve a number of less dramatic motions of the biopolymer's sidechains and even more subtle rearrangements of water molecules around the biopolymer. Each of these correspond to transitions between small free energy minima, the intricate sequences of which form the overall backbone movements. Thus, it could be oversimplifying to describe the backbone transitions as "crossing" some energy barriers in the processes of changing the fluctuation areas on the Ramanchandran plots (Fig. 3).

From the other hand, the dynamics within the local minima on the "funnel" is believed to be random, that is equivalent to the purely diffusive motion. Thus, the way the trajectory explores the space of available conformations has to be a random search process. It is, therefore, very important to check this assumption, because, if it is not the case, that would mean that there exists a non-trivial "flow" of trajectories. Such flow would imply that the system explores the conformational space in a more "intelligent" self-organised manner.

The dynamical investigation of protein systems is a challenging task. Among the few published works there are attempts to analyse the chaoticity of the dynamics calculating the Lyapunov exponents of the molecular trajectory [4]. Another important study in this direction shows an apparent non-ergodicity of the protein trajectories during the process of crossing the barrier between two minima in a simple protein model system $[5,6]$. The non-ergodicity is caused by different trajectory separation rate before and after the transition 
and may cause the "non-random" character of the conformational search in proteins. We have recently found the hidden non-randomness in molecular trajectories: a highly non-uniform covering of the molecular phase space in strong contradiction to the assumption of the random ergodic exploration of the phase space $[7,8]$. For peptides in explicit water there are long periods (dozens of nanoseconds) when the molecule is at "dynamical frustration", that is it does not explore other configurations [9]. Here non-ergodicity plays a critical role because it significantly modifies the transition rates between the minima.

In this study we make an attempt to directly investigate the dynamics in the transitions and between them. We have chosen the "middle section" of the "funnel" when the initial quick collapse of the molecule has occurred and first secondary structural motifs start forming. For a typical peptide this is the stage in the folding process where the system spends most of the time. We investigate the dynamics of the system keeping it on the same energy surface which is possible to do since we simulate the system using molecular dynamics (MD). By introducing minimal perturbations we misplace the system from its original trajectory and analyse the implications to the transitions and the dynamics between them. Particular attention has been given to the role of water in the process.

We have found that the majority of the trajectories between the transitions, where the system spends most of the time, lead to the transitions, thus implying a self-coordination of the system's atoms. However, the actual short process of the transition is extremely sensitive to the perturbations. A minute displacement of the trajectory prevents the transition. Most intriguing is that this is true for any atom of the system, including the water molecules very distant from the part of the peptide under transition.

\section{Molecular system and method}

A 21-residue peptide A5(A3RA)3A, that is known to fold in $0.8 \mu \mathrm{s}$ [10], was MD simulated in explicit water with the time step of $2 \mathrm{fs}$. The initial conformation of the molecule was taken after a $100 \mathrm{~ns}$ run started from the streched configuration. At this moment an $\alpha$-helix has partially formed. The forcefield for the simulations was GROMOS96 [11]. The peptide was solvated by 1658 SPC water molecules [12] and after a proper minimisation of the system's energy and initial equilibration was simulated for a total of $50 \mathrm{~ns}$ using the GROMACS molecular dynamics [13] package. The temperature and the pressure of the system were kept constant at $300 \mathrm{~K}$ and 1 bar respectively using Berendsen [14] thermostat. 


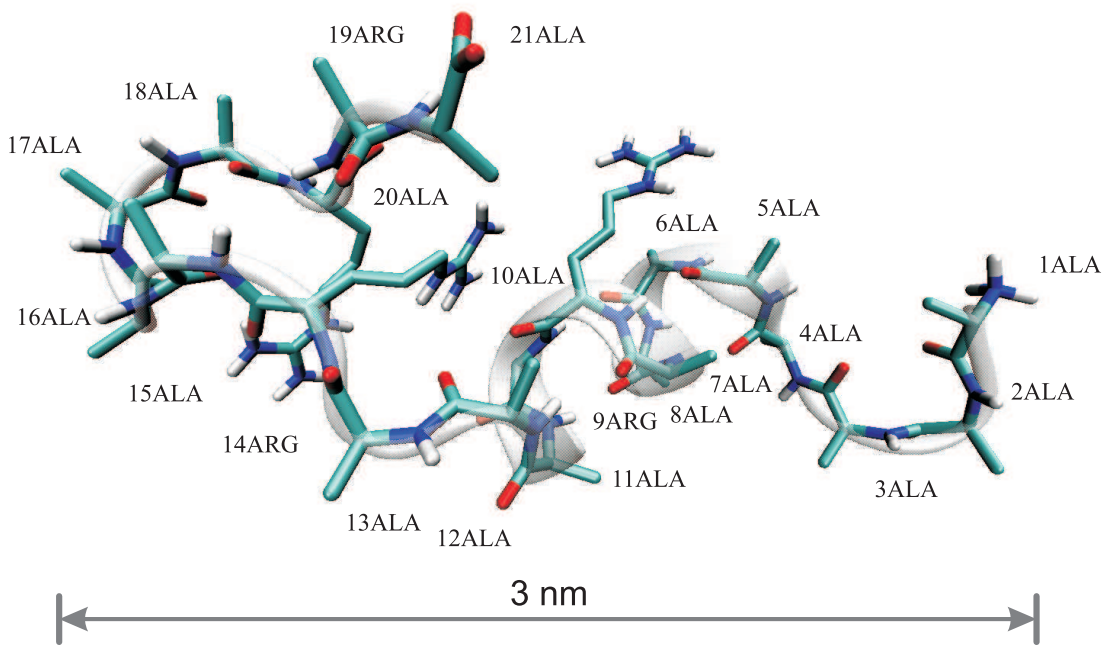

Fig. 1. The A5(A3RA)3A molecule used in the study. Carbon atoms are light blue, oxygens are red, nitrogens are dark blue, and hydrogens are grey. The united atoms force field $53 \mathrm{a} 6$ was used. The backbone motifs are depicted in light grey

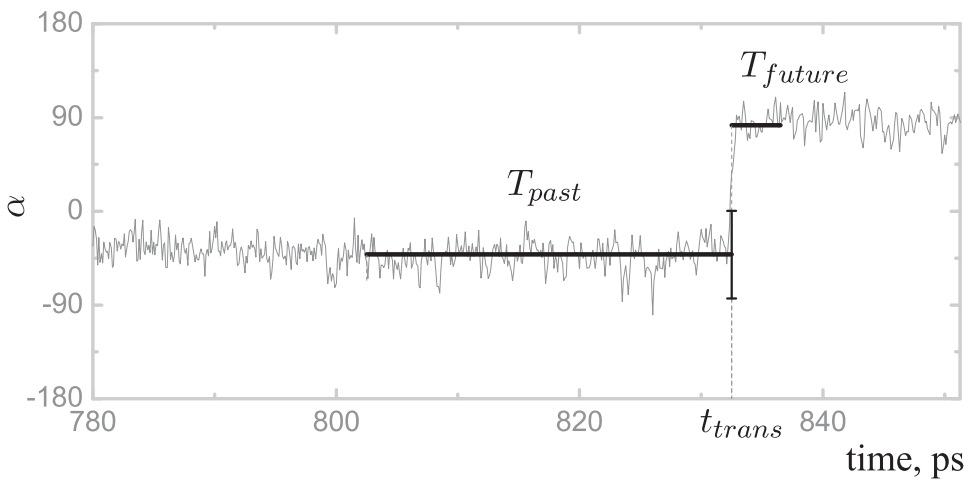

Fig. 2. The definition of the transition in peptide's configuration used to find the transition moments and dihedral angles involved. For each dihedral angle $\alpha$ of the peptide (in this example the $\psi$ angle of the residue 2ALA was taken) the transition time $t_{\text {trans }}$ is defined as the time when the mean of $\alpha$ in the interval $T_{\text {future }}$ is outside the range $\langle\alpha\rangle \pm 3 \sigma_{\alpha}$, where $\langle\alpha\rangle$ is the mean of $\alpha$ in the interval $T_{\text {past }}$ and $\sigma_{\alpha}$ is the standard deviation for the same set of values. $T_{\text {future }}$ and $T_{\text {past }}$ were equal to 4 ps and 30 ps respectively

A typical conformation of the molecule in the simulations is shown in Fig. 1. An $\alpha$-helix (residues 4 to 12) and a turn (residues 15 to 18) remain stable for the period of the simulations. We would like to stress that the present study focuses on the elementary transitions, therefore the statistical representation of the results is based on the number of transitions analysed rather than the total simulation length. The transitions in the peptide's conformation were defined as described in Fig. 2. In the original (unperturbed) run there were a total of 76 transitions over the period of $6 \mathrm{~ns}$, that is on average one transition per 79 ps. The dihedral angles involved in the transitions are shown in Fig. 3. 
The dynamics is investigated by introducing perturbations into the system. The perturbations were minimal: the velocity of only one atom of the system was randomly rotated by a few degrees. This keeps the system on the same energy surface since both the coordinates of the atoms and the magnitude of their velocities remain unchanged. Two protocols of such perturbations were used.

I. Periodic perturbations with the period from $\Delta t=8$ fs to $100 \mathrm{ps}$. At the time moments $t=i \Delta t, i=0 . . N$, where $N$ is the total number of steps in the simulation a randomly chosen peptide's atom was perturbed.

II. One perturbation just before each transition. At the times $t_{\text {trans }}$ a randomly chosen atom of the dihedral angle that undergoes the transition (or of a randomly chosen water molecule, see section 3) was perturbed. The part of the trajectory after the time $t_{\text {trans }}$ was discarded and the simulation was restarted from time $t_{\text {trans }}$ with exactly the same system state except for the perturbed atom. This procedure resulted in the interruptions of the trajectory at every $\approx 14 \mathrm{ps}$.

\section{Results and Discussion}

The dynamics of three representative dihedral angle pairs are shown in Fig. 3. Two pairs (5ALA and 19ARG) exhibit two and three transitions respectively, while the third one (7ALA) fluctuates in the same conformation. The latter one belongs to the $\alpha$-helix that explains its stable dynamics during the simulation. The rest of the aminoacids sum up to 76 transitions (shown at the bottom of Fig. 3). As it can be seen from the figure, the transition itself is relatively fast compared to the periods of stable fluctuations. Typically, a transition takes from 10 to 15 ps. Most of the transitions involve only one residue, however, about $25 \%$ of them involve two to five neighbouring (not necessarily adjacent) residues.

Perturbations of the two types described above have very different effects on the behaviour of the system. We find that despite a very small perturbation it is possible to keep the molecule in the same dynamic regime (Fig. 4) without entering conformational transitions if the perturbation is introduced just before the transition (protocol II). The way the perturbations are introduced prevents the peptide from making the transition, that is the trajectory can make several "attempts" for the transition before the simulation can progress for longer times. Most of the times only one perturbation is enough, however for some transitions several attempts are required to prevent the transition, see Fig. 4. There are few transitions that require a significant number (50-60) of attempts. Such transitions appear with the period of approximately $1 \mathrm{~ns}$. It 


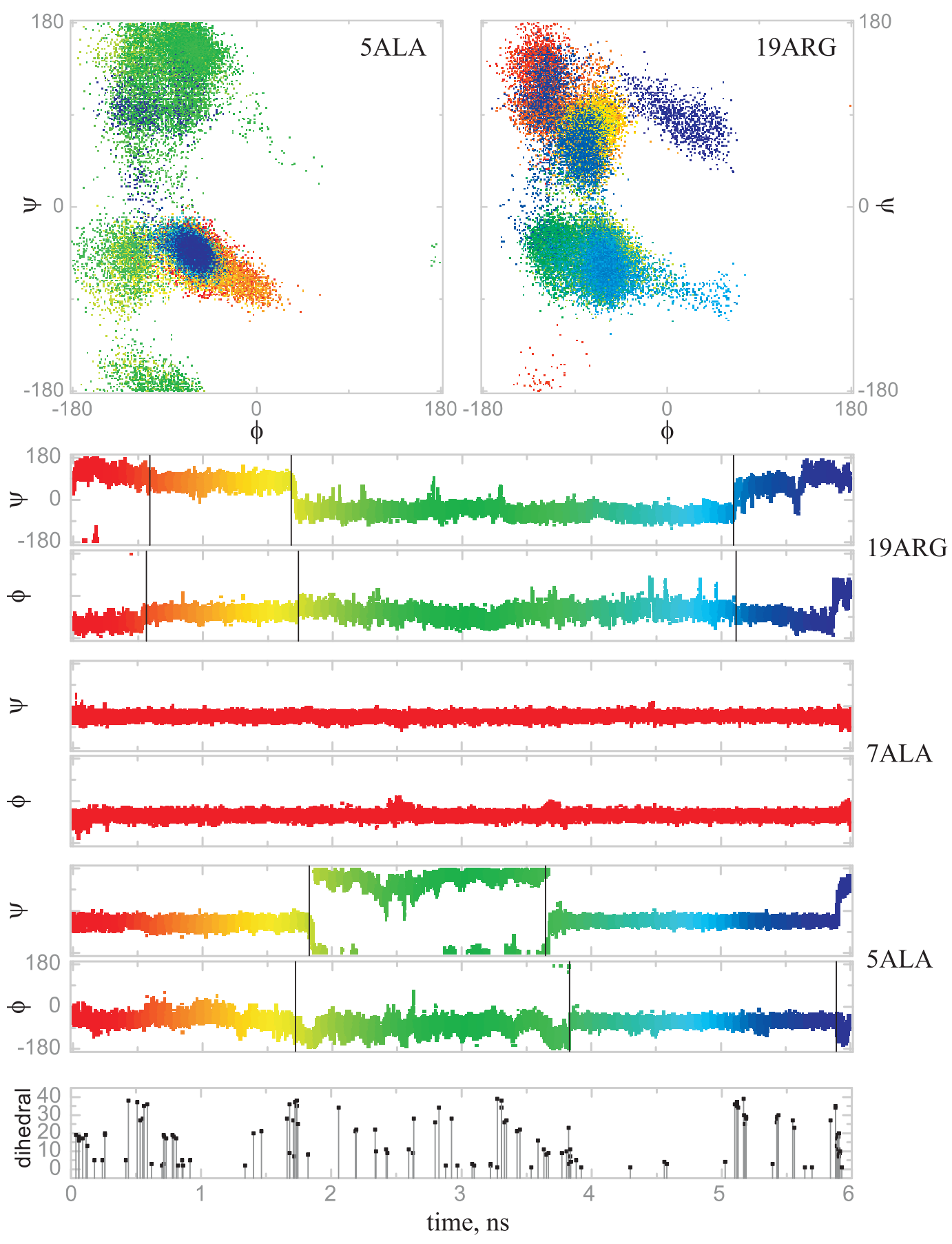

Fig. 3. The dynamics of three representative dihedral angle pairs of the molecule A5(A3RA)3A and corresponding Ramachandran plots. The colouring on the time plots and the Ramachandran plots are the same and depict different time moments of the simulation. Clear separation of the fluctuation areas are visible on the Ramachandran plots. At the bottom all the transitions are shown as the dihedral angle index involved in the transition 

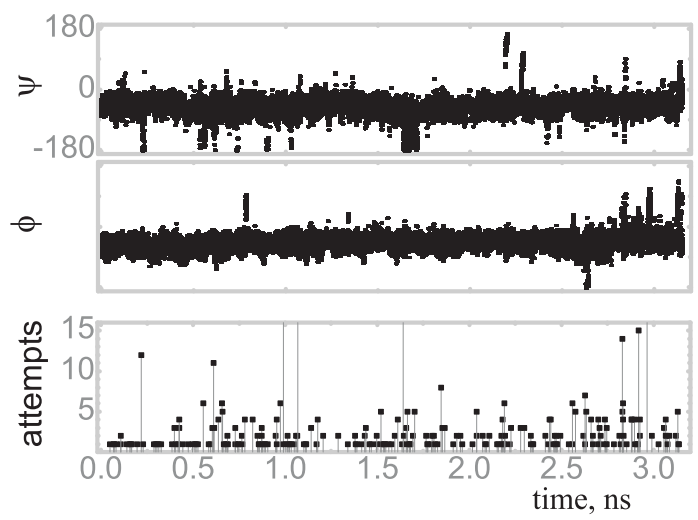

Fig. 4. The dynamics of the dihedral angles of the residue 19ARG perturbed using protocol II (see section 2). The number of "attempts" needed to prevent the transitions are shown at the bottom (see section 3 )

should be stressed that not the same dihedral angle undergoes a transition at different attempts at one transition time. Typically, 3-5 angles and even more for the "many attempts" transitions undergo the conformational changes. This implies, that there are many different "exit channels" from the basin of stable fluctuations that are found by the trajectory surprisingly fast (on the average with the $\approx 14$ ps period).

Unexpectedly, it seems that the phenomenon does not depend on which atom's velocity is perturbed. We have tested atoms of the residue under transition, other residues, and water atoms including the waters that are several water diameters away (up to the simulation box size, $3.7 \mathrm{~nm}$ ) from the peptide (see Fig. 1 for the scale). The overall behaviour is very similar to what is observed for the one residue perturbations: for water perturbations the average time between the transitions is 16ps and the distribution of the "attempts" is very similar to what is shown in Fig. 4.

Because of such sensitivity to perturbations it would be reasonable to assume that periodic perturbations of the same nature (protocol I) would have the same effect on the system. However, we have found that periodic perturbations do not change the number of the transitions significantly (although they, as expected, occur at different time moments). There were 51 and 54 transitions for the perturbations with the periods of 100ps and 1ps respectively, Fig. 5. Moreover, even much stronger perturbations when all peptide's atoms or all water atoms are perturbed every 8 fs did not prevent the transitions. Only when the coordinates of the atoms in addition to the velocities were displaced, the number of transitions dropped significantly. This, however, implies not only the change of the trajectory but also jumps between different potential surfaces.

To rationalise the effect (or rather it's absence) of the periodic perturbations it is necessary to conclude that away from transitions most of the neighbouring 


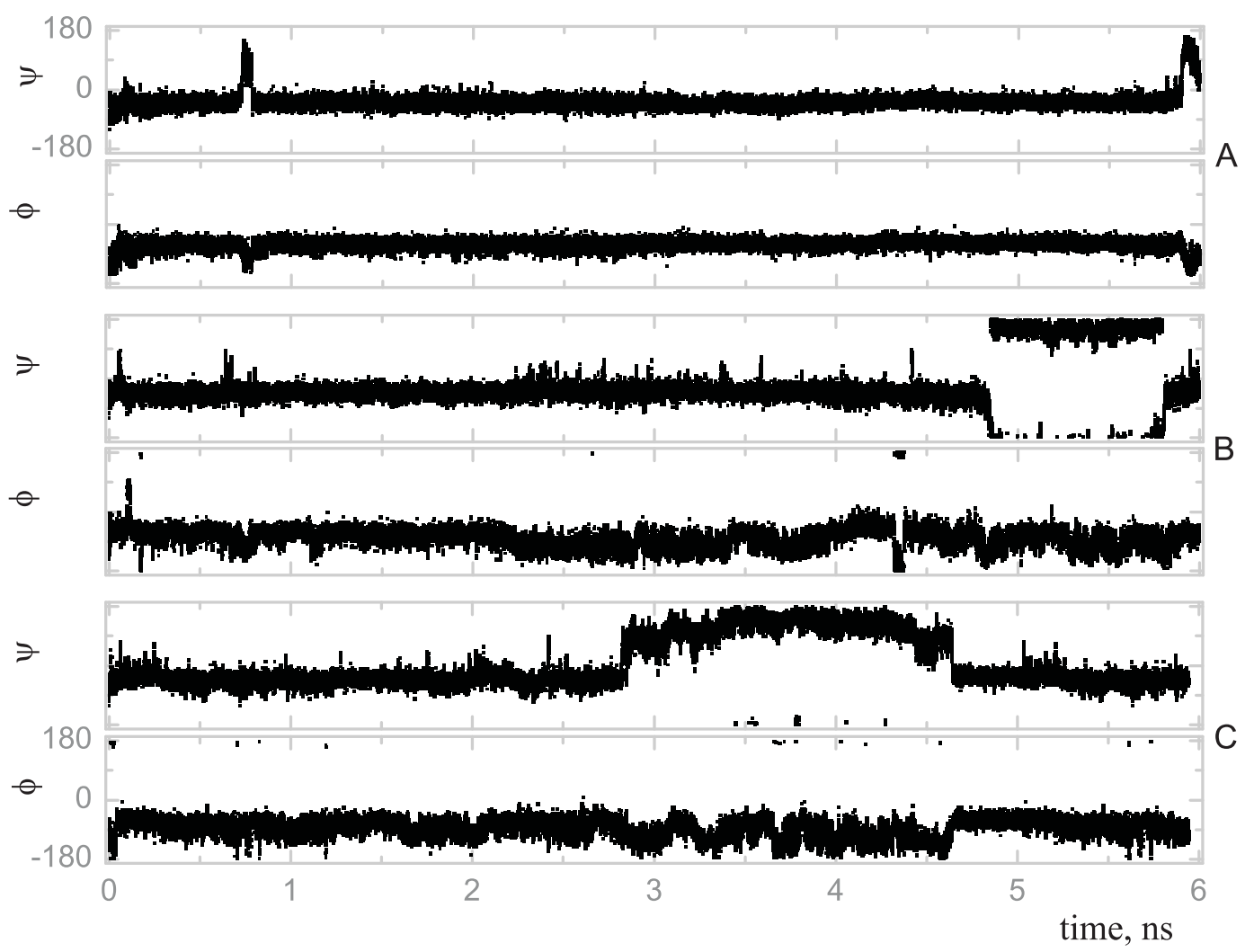

Fig. 5. The dihedral angles of the residue 10ALA are shown in different simulations: A - original (unperturbed), B - periodic perturbation, 100 ps period, C - periodic perturbation, 1 ps period

trajectories (the phase space flow) are aligned in the same direction - towards the transition. That is why jumping from one trajectory to the other would still lead to the transition. It should also be noted that the timescale between the transitions is longer than the correlation time in bulk water (less than few picoseconds at normal conditions). Therefore, this also indirectly indicates the existence of longer correlations in the peptide's motion.

On the contrary, the dynamical regime at the moment of transition is very different. Here close trajectories do not necessary belong to the "transition channel" and minute perturbations result in qualitatively different behaviour of the system. From the molecular point of view this means that at the transitions the atoms are involved in strongly collective motion and disrupting one atom has a dramatic effect. Most interestingly, water atoms are as important in this motion as the atoms of the peptide itself. 


\section{Conclusions}

Summarising, the dynamics of the peptide probed by minimal perturbations of molecular trajectories that keep them on the same energy surface is very different at the short periods of structural transitions compared to the regime of fluctuations between the transitions. The latter is characterised by a robust dynamics when neighbouring trajectories all lead to transitions with similar probability. Because the average time between the transitions is appreciably longer than the autocorrelations in water this indicates that collectively the peptide motion posses longer scale time correlations.

The dynamics during the transition processes is very different. It is very sensitive to the perturbations and the peptide can easily be prevented from the transition. Most interestingly, the perturbations of atoms different from the residue involved in the transition and even distant water atoms have the same effect on preventing the transitions. This shows that the dynamics during the transitions is very collective, involving all atoms in the system.

Acknowledgements. The work is supported by Unilever and the European Commission (EC Contract Number 012835 - EMBIO).

\section{References}

[1] S. Hovmoller, T. Zhou, T. Ohlson, Conformations of amino acids in proteins, Acta Crystallographica Section D 58 (5) (2002) 768-776.

URL

http://www.blackwell-synergy .com/doi/abs/10.1107/S0907444902003359

[2] P. Banushkina, M. Meuwly, Diffusive dynamics on multidimensional rough free energy surfaces, The Journal of Chemical Physics 127 (13) (2007) 135101.

URL http://link. aip.org/link/?JCP/127/135101/1

[3] B. Alakent, P. Doruker, M. C. Camurdan, Time series analysis of collective motions in proteins, The Journal of Chemical Physics 120 (2) (2004) 10721088.

URL http://link.aip.org/link/?JCP/120/1072/1

[4] M. Braxenthaler, R. Unger, D. Auerbach, J. A. Given, J. Moult, Chaos in protein dynamics, Proteins: Structure, Function, and Genetics 29 (4) (1997) $417-425$.

[5] K. Matsumoto, Dynamics of protein near transition between local minima, Physics Letters A 298 (2-3) (2002) 117-121. 
[6] K. Matsumoto, Non-ergodicity induced by isotropic random perturbation, Chaos: An Interdisciplinary Journal of Nonlinear Science 13 (3) (2003) 10261031.

URL http://link.aip.org/link/?CHA/13/1026/1

[7] D. Nerukh, V. Ryabov, R. C. Glen, Complex temporal patterns in molecular dynamics: a direct measure of the phase space exploration by the trajectory at macroscopic time scales, Physical Review E 77 (2008) 036225.

[8] D. Nerukh, Computational mechanics reveals nanosecond time correlations in molecular dynamics of liquid systems, Chemical Physics Letters 457 (4-6) (2008) 439-443.

[9] D. Nerukh, Dynamical frustration of protein's environment at the nanoseconds time scale, J. Mol. Liq. in press.

[10] J. Kubelka, J. Hofrichter, W. A. Eaton, The protein folding 'speed limit, Current Opinion in Structural Biology 14 (1) (2004) 76-88.

[11] W. Scott, P. Hunenberger, I. Tironi, A. Mark, S. Billeter, J. Fennen, A. Torda, T. Huber, P. Kruger, W. van Gunsteren, J. Phys. Chem. A 103 (1999) 3596.

[12] H. J. C. Berendsen, J. Postma, W. van Gunsteren, J. Hermans, Interaction model for water in relation to protein hydration, in: B. Pullman (Ed.), Intermolecular Forces, D. Reidel Publishing Company, Dordrecht, 1981, pp. $331-342$.

[13] D. van der Spoel, E. Lindahl, B. Hess, G. Groenhof, A. E. Mark, H. J. C. Berendsen, Gromacs: Fast, flexible and free, J. Comp. Chem. 26 (2005) 17011718 .

[14] H. J. C. Berendsen, Transport properties computed by linear response through weak coupling to a bath, in: M. Meyer, V. Pontikis (Eds.), Computer Simulations in Material Science, Kluwer, 1991, p. 139155. 